PROCEEDINGS OF THE

AMERICAN MATHEMATICAL SOCIETY

Volume 130, Number 9, Pages 2525-2534

S 0002-9939(02)06366-9

Article electronically published on February 4, 2002

\title{
A PROOF OF PIERI'S FORMULA USING THE GENERALIZED SCHENSTED INSERTION ALGORITHM FOR RC-GRAPHS
}

\author{
MIKHAIL KOGAN AND ABHINAV KUMAR
}

(Communicated by John R. Stembridge)

\begin{abstract}
We provide a generalization of the Schensted insertion algorithm for rc-graphs of Bergeron and Billey. The new algorithm is used to give a new proof of Pieri's formula.
\end{abstract}

\section{INTRODUCTION}

RC-graphs were first defined by Fomin and Kirillov [3] and later studied by Bergeron and Billey [1]. They encode the monomials contributing to the expansion of Schubert polynomials. These polynomials were introduced by Lascoux and Schutzenberger [6], [7] and described at length by Macdonald [8] and by Manivel [9].

A central problem in the theory of Schubert polynomials is to provide effective ways of computing the generalized Littlewood-Richardson coefficients $c_{v w}^{u}$ in the expansion

$$
P_{v} P_{w}=\sum_{u} c_{v w}^{u} P_{u}
$$

where $P_{w}$ is the Schubert polynomial of a permutation $w$.

The first attempt to compute the generalized Littlewood-Richardson coefficients using rc-graphs was made in [1], where Monk's formula was proved using a generalized Schensted insertion algorithm. Later, in [5, this generalized algorithm was used to compute a more general set of Littlewood-Richardson coefficients. Unfortunately, this algorithm does not work in general and, in particular, fails to give a proof of Pieri's formula. In this paper we will modify this algorithm to inserting a whole row of elements at once instead of an element by element insertion. This generalization will prove Pieri's formula.

Let us give a statement of Pieri's formula, which was originally proved in [6] and later reproved by other methods [1], [10, 13]. Our way of stating Pieri's formula is not standard, but in Section 4 we show how to deduce the standard formulation of Pieri's formula from Theorem 4.1

\section{Theorem 4.1.}

$$
P_{w} P_{\sigma[r, m]}=\sum P_{w^{\prime}}
$$

Received by the editors November 17, 2000 and, in revised form, April 6, 2001.

2000 Mathematics Subject Classification. Primary 14N15.

(C)2002 American Mathematical Society 
where the sum is over all $w^{\prime}=w t_{a_{1}, b_{1}} \ldots t_{a_{m}, b_{m}}$ such that the $b_{i}$ 's are distinct and greater than $r, a_{1} \leq a_{2} \leq \ldots \leq a_{m} \leq r$, satisfying $l\left(w^{\prime}\right)=l(w)+m$ and $w^{\prime}\left(b_{i}\right)<$ $w^{\prime}\left(b_{j}\right)<w^{\prime}\left(a_{i}\right)$ for every $i<j$ with $a_{i}=a_{j}$.

In the above statement, $\sigma[r, m]$ stands for the permutation $[1,2, \ldots, r-1, r+$ $m, r, r+1, \ldots]$. Note that $P_{\sigma[r, m]}$ is equal to the homogeneous symmetric polynomial $h_{m}\left(x_{1}, \ldots, x_{r}\right)$.

We will define Schubert polynomials and rc-graphs, and point out their relationship, in Section 2. The generalized insertion algorithm will be given in Section 3, while in Section 4 we will prove Pieri's formula by providing the inverse algorithm.

\section{RC-GRAPHS}

Let us decide on some notation and state some basic facts about permutations and Schubert polynomials first. For a permutation $w \in S_{n}$, where $S_{n}$ is the symmetric group on $n$ elements, we can write $w$ as $[w(1), \ldots, w(n)]$. We let $t_{a b}$ denote the transposition which takes $a$ to $b$ and vice versa, leaving other elements fixed, and write $s_{i}$ for $t_{i, i+1}$. It can be shown that $s_{1}, \ldots, s_{n-1}$ generate $S_{n}$ with the relations

$$
\begin{aligned}
s_{i}^{2} & =1, \\
s_{i} s_{j} & =s_{j} s_{i} \text { if }|i-j| \geq 2, \\
s_{i} s_{i+1} s_{i} & =s_{i+1} s_{i} s_{i+1} .
\end{aligned}
$$

For a permutation $w \in S_{n}$, we let the length $l(w)$ of $w$ be the number of inversions of $w$, i.e. the number of pairs $(i, j)$ with $i<j$ and $w(i)>w(j)$. A string $a_{1} a_{2} \ldots a_{p}$ such that $s_{a_{1}} \ldots s_{a_{p}}=w$ with $p$ minimal is called a reduced word for $w$. It can be shown that this $p$ is just the length of $w$, and any two reduced word decompositions can be transformed into each other using the last two relations given above. Denote by $R(w)$ the set of all the reduced words for $w$.

This enables us to define Schubert polynomials. Let $\partial_{i}$ be an operator which, acting on a polynomial $f\left(x_{1}, \ldots, x_{n}\right)$ to the left, yields the polynomial

$$
\frac{f\left(x_{1}, \ldots, x_{i}, x_{i+1}, \ldots, x_{n}\right)-f\left(x_{1}, \ldots, x_{i+1}, x_{i}, \ldots, x_{n}\right)}{x_{i}-x_{i+1}} .
$$

It can be verified that

$$
\begin{aligned}
\partial_{i}^{2} & =0 \\
\partial_{i} \partial_{j} & =\partial_{j} \partial_{i} \text { if }|i-j| \geq 2, \\
\partial_{i} \partial_{i+1} \partial_{i} & =\partial_{i+1} \partial_{i} \partial_{i+1} .
\end{aligned}
$$

Thus for any permutation $w$, we may define $\partial_{w}$ as $\partial_{a_{1}} \ldots \partial_{a_{p}}$, where $a_{1} \ldots a_{p}$ is any reduced word for $w$. Now the Schubert polynomial $P_{w}$ is defined to be

$$
P_{w}=\partial_{w^{-1}} w_{0} x_{1}^{n-1} x_{2}^{n-2} \ldots x_{n-1}^{1} x_{n}^{0},
$$

where $w_{0}=[n, \ldots, 1]$ is the longest permutation in $S_{n}$.

Now we come to rc-graphs. Given a reduced word $a_{1} \ldots a_{p}$ for $w$, we say that a sequence $\alpha_{1}, \ldots, \alpha_{p}$ is compatible for this word if

$$
\begin{array}{r}
\alpha_{1} \leq \alpha_{2} \leq \ldots \leq \alpha_{p}, \\
\alpha_{i} \leq a_{i}, \forall i, \\
\alpha_{i}<\alpha_{i+1} \text { if } a_{i}<a_{i+1} .
\end{array}
$$

Denote by $C(\mathbf{a})$ the set of all compatible sequences for $\mathbf{a} \in R(w)$.

Given such a compatible sequence, we make an rc-graph by placing intersections (see Figure 1 ) at all the positions $\left(\alpha_{k}, a_{k}-\alpha_{k}+1\right)$; these form a subset of $\{1,2, \ldots\} \times$ 


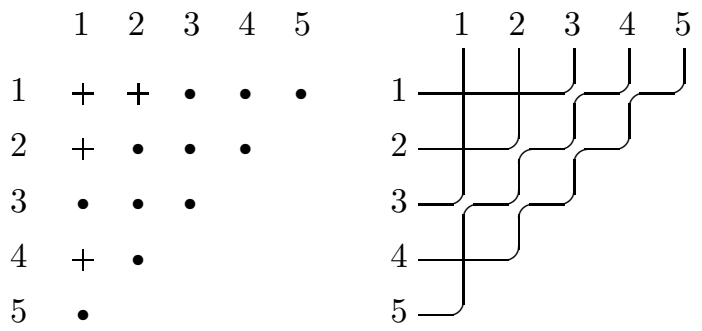

FiguRE 1. Two ways of drawing an rc-graph.
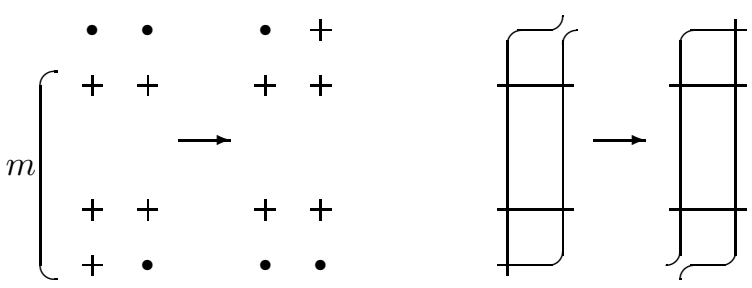

Figure 2. Ladder moves.

$\{1,2, \ldots\}$. Now we draw strands starting at each row and winding their way up. Wherever there is an intersection symbol, we make the two strands there intersect. It is easy to show by induction that the strand starting at the $i^{\text {th }}$ row ends up at the $w(i)^{t h}$ column and that no two strands intersect twice. Conversely, a graph with intersections, such that no two strands intersect twice, immediately gives us a reduced word for the permutation and also a compatible sequence (we look at the intersections in order of increasing row number; going from right to left in each row, the row number gives the $\alpha_{i}$, and to get $a_{i}$ we add the row number and column number and subtract 1). For more details see 1 .

Denote by $\mathcal{R C}(w)$ the set of all rc-graphs which correspond to $w$. For $D \in \mathcal{R C}(w)$ let $x^{D}=\prod_{\left(\alpha_{k}, a_{k}-\alpha_{k}+1\right) \in D} x_{\alpha_{k}}$. Now we state a theorem proved in [2] and in [4].

Theorem 2.1. For any permutation $w \in S_{\infty}$,

$$
P_{w}=\sum_{\mathbf{a} \in R(w)} \sum_{\alpha_{1} \ldots \alpha_{p} \in C(\mathbf{a})} x_{\alpha_{1} \ldots x_{\alpha_{p}}}=\sum_{D \in \mathcal{R} \mathcal{C}(w)} x^{D} .
$$

On an rc-graph we can define a ladder move (of size $m$ ) as shown in Figure 2. Note that after the move we get an rc-graph corresponding to the same permutation (since the number of intersections is preserved and the same strands intersect in the two figures).

We shall call the inverse operation an inverse ladder move. Billey and Bergeron in [1] prove that all graphs in $\mathcal{R C}(w)$ can be obtained by applying a succession of ladder moves to $D_{b o t}(w)=\left\{(i, c): c \leq m_{i}\right\}$ (where $m_{i}=\#\{j: j>i$ and $\left.w(j)>w(i)\}\right)$, which is the "bottom" rc-graph corresponding to $w$. Note that the bottom graph has the property that the rows are left-justified. For instance, the graph shown in Figure 1 is a bottom graph. 


\section{GenERALIZING THE INSERTION ALGORITHM}

Recall that Pieri's rule seeks to give a formula for multiplication of a Schubert polynomial by $P_{\sigma[r, m]}$ (a homogeneous symmetric polynomial). In other words, it computes the generalized Littlewood-Richardson coefficients $c_{v w}^{u}$ for $w=\sigma[r, m]$, where the coefficients are defined by

$$
P_{v} P_{w}=\sum_{u} c_{v w}^{u} P_{u}
$$

(We use the fact that Schubert polynomials form a basis of all polynomials to get a unique such expansion.) To enable us to find this formula, we need a modification of the insertion algorithm, which was defined in [1] and used to compute a family of Littlewood-Richardson coefficients in [5].

Notice that each rc-graph for the permutation $\sigma[r, m]$ can be given by specifying the number of intersections in the rows on or above row $r$. Assume this rc-graph is given by $k_{s}$ elements in row $i_{s}$, where $r \geq i_{1}>i_{2}>\ldots>i_{t}>0$ and $\sum k_{s}=m$. Below is the algorithm which inserts this rc-graph into a general rc-graph.

Algorithm. Given an rc-graph $D$, and a level $r$, suppose we have to insert $k_{s}$ elements into row $i_{s}$ of $D$, where $r \geq i_{1}>i_{2}>\ldots>i_{t}$. We keep an ordered sequence of $\left(a_{i}, b_{i}\right)$ 's during the course of the algorithm, such that $a_{1} \leq a_{2} \leq \ldots \leq a_{l} \leq r$, each $b_{i}>r$ and all $b_{i}$ 's are distinct. Initially the sequence is empty.

Proceed row by row as follows. Starting with row $i_{1}$, find the rightmost position $\left(i_{1}, j\right)$ where the configuration is one of the following:

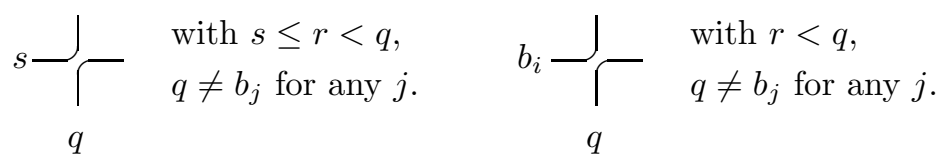

FiguRE 3 .

Add this intersection. If we are in the first case, insert $(s, q)$ into the sequence $\left(a_{i}, b_{i}\right)$ in the rightmost position, such that $a_{i}$ 's remain nondecreasing in the sequence. $((s, q)$ are the rows where the two strands shown in Figure 3 originate.) If we are in the second case, add $\left(a_{i}, q\right)$ just before where $\left(a_{i}, b_{i}\right)$ is in the sequence. Repeat the above process $k_{1}$ times to insert $k_{1}$ intersections into the row $i_{1}$ to get a new graph together with a sequence $\left(a_{1}, b_{1}\right), \ldots,\left(a_{k_{1}}, b_{k_{1}}\right)$ where we know for sure that the $b_{i}$ 's are distinct and greater than $r$ and the $a_{i}$ 's are ordered and less than or equal to $r$. Also, the graph we have obtained might not be an rc-graph. So we go up to the next row and perform the following rectification procedure. Starting from the left, we look for either of the two configurations in Figure 4.
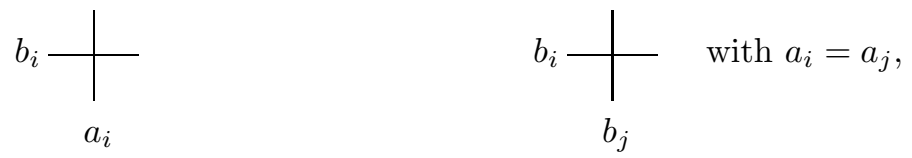

FigURE 4. 
We remove the intersection, and delete the pair $\left(a_{i}, b_{i}\right)$ from the sequence of $(a, b)$ 's. If the intersection was at position $(i, j)$, find the maximal $j^{\prime}<j$ such that the configuration at $\left(i, j^{\prime}\right)$ is of the form shown in Figure 3. (Such a position must exist, since the $b_{i}$ is greater than $r$ and $\left(a_{i}, b_{i}\right)$ is no longer in the sequence.) We add the intersection, and to the list of $(a, b)$ 's we add the new pair the same way it was described before. Then we look to the right to see if there is another intersection of the form given in Figure 4. If it exists, remove it together with the appropriate $\left(a_{i}, b_{i}\right)$ and proceed as before. We call this process rectification of a given row.

When we are done with rectifying the current row, we add what intersections we must to this row in the same manner as for the previous row, giving us some new pairs $\left(a_{j}, b_{j}\right)$. We then proceed to the next row and repeat the procedure. It is clear from the construction that the $b$ 's remain distinct and the $a$ 's remain ordered.

The rest of this section is concerned with proving the following:

Theorem 3.1. The above algorithm produces an rc-graph.

Before proving Theorem 3.1 we will need two lemmas and another algorithm.

Lemma 3.2. After row $\ell$ has been rectified, the strands $b_{i}, \ldots, b_{i+k}$ with $a=a_{i}=$ $\ldots=a_{i+k}$ pass from row $\ell$ to row $\ell-1$ to the left of the place where the strand $a$ passes from row $\ell$ to row $\ell-1$. Moreover, they pass from row $\ell$ to row $\ell-1$ in the same order they appear in the $(a, b)$ sequence. (See Figure 5 for an illustration.)

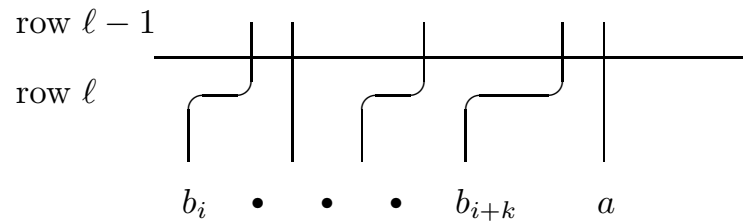

FIGURE 5. The order in which the strands $b_{i}, \ldots, b_{i+k}$ and $a$ intersect the line, which separates rows $\ell$ and $\ell-1$, is the same as the order of $b_{i}$ 's in the $(a, b)$ sequence.

Proof. This lemma can be proved by induction on $\ell$. At the beginning of the algorithm, set $\ell=r-1$. Then the statement of the lemma holds, since the $(a, b)$ sequence is empty.

Assume we know that the lemma holds for $\ell+1$. Notice that if during the rectification of row $\ell$ no pairs $\left(a_{j}, b_{j}\right)$ with $a_{j}=a$ were added or removed from the $(a, b)$ sequence, the strands $b_{i}, \ldots, b_{i+k}$ and $a$ never intersect each other in row $\ell$. Hence they pass between rows $\ell$ and $\ell-1$ in the same order they pass from row $\ell+1$ to row $\ell$.

Moreover, the order of the strands $b_{i}, \ldots, b_{i+k}$ and $a$ can change only when some of them intersect in row $\ell$. But then this intersection is one of the intersections in Figure 4. The algorithm removes this intersection, and it removes the appropriate tuple from the $(a, b)$ sequence to preserve the order of the stands.

At the same time, when an intersection is added, the addition of $\left(a_{j}, b_{j}\right)$ to the list is consistent with order of the strands $b_{i}, \ldots, b_{i+k}$ and $a$ passing from row $\ell$ to row $\ell-1$. 
Lemma 3.3. The permutation carried by the strands of the graph at each moment of the algorithm is $w t_{a_{1} b_{1}} \ldots t_{a_{m^{\prime}} b_{m^{\prime}}}$ where $\left\langle\left(a_{1}, b_{1}\right), \ldots,\left(a_{m^{\prime}}, b_{m^{\prime}}\right)\right\rangle$ is the sequence up to that point in the algorithm.

Proof. We again use induction: we need to show that the above statement remains true when we insert an intersection or delete one.

Assume we are in the first case of Figure 3 ; that is, we are adding an intersection and $(s, q)$ to the list of $(a, b)$ 's. We can easily see that adding this intersection multiplies the permutation of the graph by $t_{s, q}$ on the right. So, if $\left\langle\left(a_{1}, b_{1}\right), \ldots,\left(a_{m^{\prime}}, b_{m^{\prime}}\right)\right\rangle$ is the list before this insertion, the permutation of the graph after the insertion is $w t_{a_{1} b_{1} \ldots} \ldots t_{a_{m^{\prime}} b_{m^{\prime}}} t_{s, q}$. Using the fact that $t_{a b}$ and $t_{c d}$ commute when $a, b, c, d$ are all distinct, we can commute $t_{s, q}$ trough $t_{a_{1} b_{1} \ldots} \ldots t_{a_{m^{\prime} b_{m^{\prime}}}}$ to the left until some $a_{j} \leq s$. Then the permutation carried by our graph is $w t_{a_{1} b_{1}} \ldots t_{a_{j} b_{j}} t_{s q} \ldots t_{a_{m^{\prime}} b_{m^{\prime}}}$. So, after inserting $(s, q)$ into the list after $\left(a_{i}, b_{j}\right)$, the property we are trying to prove holds.

If we are in the second situation of Figure 3, then we multiply the permutation $w t_{a_{1} b_{1} \ldots} \ldots t_{a_{m^{\prime}} b_{m^{\prime}}}$ by $t_{b_{i}, q}$ on the right. Then $t_{b_{i}, q}$ can be moved to the left until the place $i$, since all $b_{j}$ 's are distinct and thus $t_{b_{i}, q}$ commutes with all $t_{a_{j}, b_{j}}$ if $j>i$. After that we use the following identity, which shows why we have to add $\left(a_{i}, q\right)$ to the $(a, b)$ list before $\left(a_{i}, b_{i}\right)$ :

$$
t_{a, b_{i}} t_{b_{i}, q}=t_{a, q} t_{a, b_{i}} .
$$

For the deletion, we use almost identical arguments in both cases of Figure 4, since the deletion of an intersection also multiplies the permutation of the graph by the appropriate transposition. The only difference is that in the second case, instead of (3.1) we have to use the following identity:

$$
t_{a, b_{i}} t_{a, b_{j}} t_{b_{i}, b_{j}}=t_{a, b_{j}} .
$$

This concludes the proof of the lemma.

To give a proof of Theorem 3.1 we have to introduce another algorithm (call it Algorithm 2) that we perform on the intermediate graph. This algorithm will take the graph which was rectified upto the row $\ell$, and remove some intersections to produce an rc-graph of the original permutation $w$.

Algorithm 2. We start with the list of $\left(a_{i}, b_{i}\right)$ 's and, starting with the last row $\ell$ we have finished with, go down row by row and from right to left in each row. We look for intersections of the form
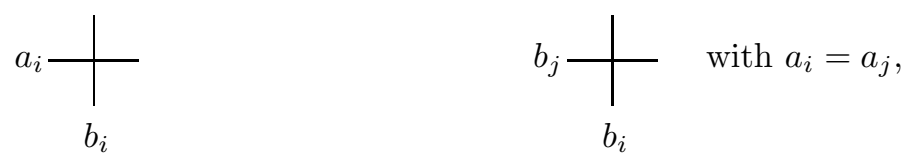

FigURE 6.

Whenever we see these, we remove the intersection and $\left(a_{i}, b_{i}\right)$ from its place in the list. Note that Lemma 3.2 and Lemma 3.3 hold at each moment of this algorithm. Ultimately all the $\left(a_{i}, b_{i}\right)$ will be removed from the list because each strand $a_{i}$ definitely intersects the strand $b_{i}$ at each moment of Algorithm 2 by Lemma 3.2 so all of them will get removed and at the end we will get a graph and an empty list of $(a, b)$ 's. 
The result of Algorithm 2 will be a graph with the permutation $w$ by Lemma 3.3, with exactly $l(w)$ intersections. Therefore it will be an rc-graph for $w$. We will use this fact to prove that the main algorithm generates an rc-graph.

We come back to the proof of Theorem [3.1. Suppose we have rectified some row $\ell$ and inserted some elements in it, and we want to show there are no double intersections below (and including) that row. Because of the inductive hypothesis, this works for the previous row $\ell+1$ (the base case is clear). Hence we need to check that no two strands intersect at row $\ell$ and at some lower row after the algorithm has rectified row $\ell$ and inserted all the required intersections into it.

We will show that the only double intersection which might be introduced in row $\ell$ by insertions in the lower rows will be removed by the algorithm and no new double intersections will be introduced in this row.

Let us show that after rectifying row $\ell+1$, and deleting all the intersections from Figure 4 in row $\ell$ at once, there are no strands which intersect twice below row $\ell$. For this apply Algorithm 2 to the graph constructed after rectifying row $\ell+1$. Then the resulting graph is an rc-graph $D^{\prime}$ of the permutation $w$. Start applying the inverse Algorithm 2 to this rc-graph $D^{\prime}$; that is, start adding intersections to $D^{\prime}$ in the order opposite to the order they were removed. It is then not difficult to see that since we are only adding intersections from Figure 6 and at each moment there are no double intersections below row $\ell+1$, the only double intersections at row $\ell$ have to look like the ones shown in Figure 4 . This shows that by removing intersections from row $\ell$ we remove all the double intersections below or at this row.

Now let's check that we do not create any double intersections below or at row $\ell$ during rectification of this row or during the insertion of new intersections into it. Clearly the only way we could have done this is if we inserted an intersection in the position as in the second case of Figure 3, so that the strands $b_{i}$ and $q$ intersected before, in a lower row. (In the first case of Figure 3, the two strands could not have intersected in a lower row, since $s<q$.) So, we shall show that in the second case the strands $b_{i}$ and $q$ cannot intersect in a lower row, that is, $b_{i}<q$. Proving this will finish the proof of Theorem 3.1

After row $\ell$ is rectified, we again apply Algorithm 2. Notice that this algorithm keeps moving strand $b_{i}$ to the left in row $\ell$ till the last point, where $\left(a_{i}, b_{i}\right)$ is removed from the list of $(a, b)$ 's, at which point strand $b_{i}$ moves right in row $\ell$, even to the right of its original position. For example, in Figure 7 , the circled intersections are removed during Algorithm 2. When the first one is removed, $\left(a_{j}, b_{j}\right)$ is removed from the list and $b_{i}$ moves from $x$ to the left, to $z$, and when the second intersection is removed, $\left(a_{i}, b_{i}\right)$ is removed and $b_{i}$ moves to the right of $x$, to $y$.

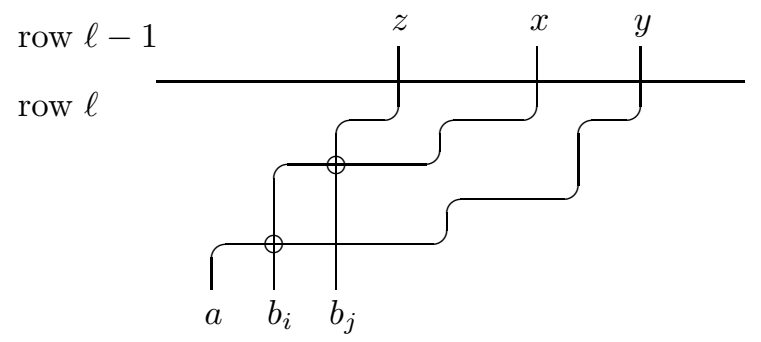

Figure 7. During Algorithm $2, b_{i}$ moved from $x$ to $z$ and then to $y$ in row $\ell$. 


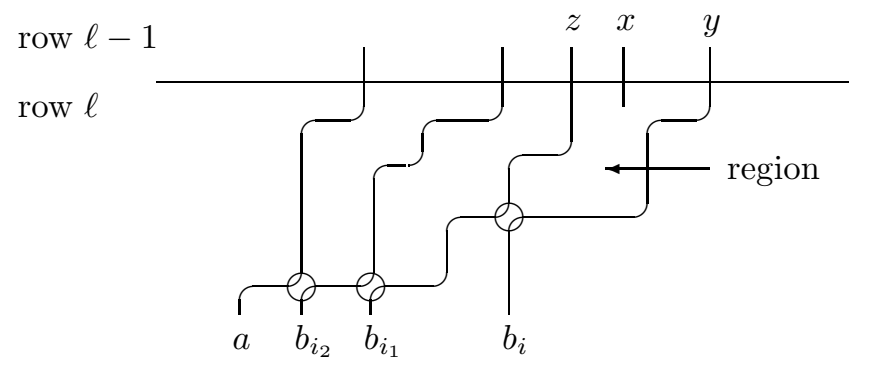

FiguRE 8 . No strands between $a$ and $b_{i}$ can pass in the shown region.

Therefore the position $x$ of strand $b_{i}$ at row $\ell$ at the start of Algorithm 2 is to the left of the position $y$ at row $\ell$ at the end of it.

Now we look at what happens after $\left(a_{i}, b_{i}\right)$ is moved off the list in Algorithm 2, by removing an intersection with $b_{i_{1}}$ (it moved first from $x$ to $z$ and then from $z$ to $y$ in row $\ell$ ). We look next at the history of $b_{i_{1}}$ till $\left(a_{i_{1}}, b_{i_{1}}\right)$ is moved off the list, and so on, getting a figure like Figure 8.

Note that no two consecutive strands shown in the figure can intersect above the encircled spot (where one of them is moved off the list), because that intersection would then have to be removed earlier than the encircled one, by the construction of Algorithm 2. Therefore, from the fact that the outcome of Algorithm 2 is an rc-graph, we conclude that in the region shown, there can pass no strand between $a$ and $b_{i}$ (otherwise some double intersections must occur). Hence the $q$ from the second case of Figure 3 must always be greater than $b_{i}$. This concludes the proof of Theorem 3.1

Remark 3.4. Assume that the permutation $w$ satisfies the condition $w(i+1)>w(i)$ if $i>r$. Each rc-graph of such a permutation has the following defining property: no two strands which start below row $r$ intersect. In this case it can be shown that the above algorithm is equivalent to one by one insertion. This is consistent with the results in [5], where the Pieri formula in this special case was proved using one by one insertion. The above statement and the fact that one by one insertion does not work in general indicate that this algorithm provides the proper generalization of the Bergeron-Billey algorithm of [1].

\section{The Proof of Pieri's formula}

To prove Pieri's formula using the above insertion algorithm, we will construct an inverse algorithm, which takes an rc-graph for $w^{\prime}=w t_{a_{1}, b_{1}} \ldots t_{a_{m}, b_{m}}$, such that the $b_{i}$ 's are distinct and greater than $r$ and $a_{1} \leq a_{2} \leq \ldots \leq a_{m} \leq r$, satisfying $l\left(w^{\prime}\right)=l(w)+m$ and $w^{\prime}\left(b_{i}\right)<w^{\prime}\left(b_{j}\right)<w^{\prime}\left(a_{i}\right)$ for every $i<j$ such that $a_{i}=a_{j}$, and produces an rc-graph for $w$ and an rc-graph for $\sigma[r, n]$ (remember that rcgraphs of $\sigma[r, m]$ are given by specifying the number of intersections $k_{s}$ in each row $s \leq r$ such that $\left.\sum k_{s}=m\right)$. Given the insertion algorithm and its inverse, we will clearly produce a bijection:

$$
\mathcal{R}(w) \times\left\{\left(k_{1}, \ldots, k_{r}\right): \sum k_{i}=m\right\}=\mathcal{R} \mathcal{C}(w) \times \mathcal{R} \mathcal{C}(\sigma[r, n]) \rightarrow \bigcup \mathcal{R}\left(w^{\prime}\right),
$$

where $w^{\prime}$ ranges as stated above. 

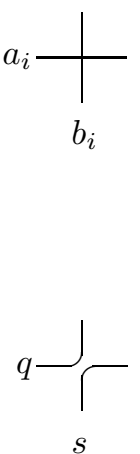

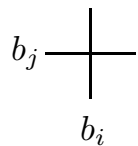

FiguRE 9.

with $s \leq r<q$, $q \neq b_{i}$ for any $i$.

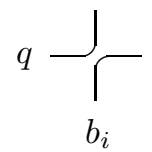

with $r<q$, $q \neq b_{j}$ for any $j$.

FiguRE 10.

Below we will produce this inverse algorithm, which will automatically prove the following theorem.

Theorem 4.1.

$$
P_{w} P_{\sigma[r, m]}=\sum P_{w^{\prime}},
$$

where the sum is over all $w^{\prime}=w t_{a_{1}, b_{1}} \ldots t_{a_{m}, b_{m}}$ such that the $b_{i}$ 's are distinct and greater than $r, a_{1} \leq a_{2} \leq \ldots \leq a_{m} \leq r$, satisfying $l\left(w^{\prime}\right)=l(w)+m$ and $w^{\prime}\left(b_{i}\right)<$ $w^{\prime}\left(b_{j}\right)<w^{\prime}\left(a_{i}\right)$ for every $i<j$ with $a_{i}=a_{j}$.

Inverse Algorithm. We are given an rc-graph $R^{\prime}$ for a permutation

$$
w^{\prime}=w t_{a_{1} b_{1}} \ldots t_{a_{m} b_{m}}
$$

with distinct $b_{i}$ 's which are greater than $r$ and with $a_{1} \leq a_{2} \ldots \leq a_{m} \leq r, l\left(w^{\prime}\right)=$ $l(w)+m$, and $w^{\prime}(a)>w^{\prime}(b)>w^{\prime}(c)$ for all $a, b, c$ such that $(a, b)$ is some $\left(a_{s}, b_{s}\right)$ and $(a, c)$ is some $\left(a_{t}, b_{t}\right)$ with $s>t$. The algorithm is defined as follows: starting from the top (first) row of the rc-graph, we look from right to left for the occurrence of one of the configurations of Figure 9.

We then remove this intersection from the rc-graph and consequently remove $\left(a_{i}, b_{i}\right)$ from the list of $(a, b)$ 's. Immediately after we remove such an intersection we look to its right for a configuration of the form shown in Figure 10.

If we do not find such a configuration, we say that an intersection is removed from this row and we move on with the algorithm, looking left along that row (and after that, going to the next row) for an intersection of the form shown in Figure 9. (At the same time we record the number of removed intersections from this row, as these numbers will give an rc-graph for $\sigma[r, m]$ at the end of the algorithm.) If we find such a configuration, we add the intersection at this place (i.e. the first configuration of this sort to the right of the removed intersection) and add $(q, s)$ or $\left(a_{i}, q\right)$ to the list of $(a, b)$ 's the same way it was done in the forward insertion algorithm, i.e we add $(q, s)$ to the $\left(a_{i}, b_{i}\right)$ 's to preserve the order of $a$ 's $\left(a_{i-1} \leq a_{i}<a_{i+1}\right)$ and we add $\left(a_{i}, q\right)$ right after $\left(a_{j}, b_{j}\right)$. Then we proceed with the algorithm, looking left for more intersections to remove. We do this till the list of $(a, b)$ 's becomes empty. At the end we are left with a graph for the permutation $w$. Moreover, the previously recorded numbers of removed intersections in each row where intersections were removed from the rc-graph produce an rc-graph for $\sigma[r, m]$. 
The fact that the resulting graph is an rc-graph can be shown using the same sort of technique used for the forward algorithm. It is also evident that the algorithm is the inverse of the forward algorithm. This finishes the proof of Theorem 4.1.

Let us now restate Pieri's formula in its original form, as it appeared in [6]. Assume that for the $(a, b)$ sequence from Theorem 4.1 we have $a_{1}=\ldots=a_{i_{1}}<$ $a_{i_{1}+1}=\ldots=a_{i_{2}}<\ldots<a_{i_{p}}=\ldots=a_{m}$. Then $w^{\prime}=w \zeta_{1} \ldots \zeta_{p}$, where each $\zeta_{k}=\left(a_{i_{k-1}+1}, b_{i_{k-1}}+1\right) \ldots\left(a_{i_{k}}, b_{i_{k}}\right)$ is a cycle. We will say that the cycle $\zeta_{k}$ has size $s\left(\zeta_{k}\right)=i_{k}-i_{k-1}$. Additionally, it follows from Theorem 4.1 that for each $\zeta_{k}$, there exists exactly one $a$ with $w(a)<w \zeta_{k}(a)$ and exactly one $a^{\prime} \leq r$ with $w\left(a^{\prime}\right) \neq w \zeta_{k}\left(a^{\prime}\right)$. (Actually, $a=a^{\prime}=a_{i_{k}}$.) Hence it is easy to conclude that Theorem 4.1 is equivalent to the following formulation of Pieri's formula given in [6].

\section{Theorem 4.2.}

$$
P_{w} P_{\sigma[r, m]}=\sum P_{w^{\prime}}
$$

where the sum is over all $w^{\prime}=w \zeta_{1} \ldots \zeta_{p}$ satisfying the following conditions: $l\left(w^{\prime}\right)-$ $l(w)=m=\sum s\left(\zeta_{k}\right)$, and each $\zeta_{k}$ is a cycle such that there exists exactly one a with $w(a)<w \zeta_{k}(a)$ and exactly one $a^{\prime} \leq r$ with $w\left(a^{\prime}\right) \neq w \zeta_{k}\left(a^{\prime}\right)$.

\section{REFERENCES}

[1] N. Bergeron and S. Billey, RC-graphs and Schubert polynomials, Experimental Math., 2 (1993), 257-269. MR 95g:05107

[2] S. Billey, W. Jockusch, R. Stanley. Some combinatorial properties of Schubert polynomials. J. Algebraic Combin. 2 (1993), no. 4, 345-374. MR 94m:05197

[3] S. Fomin and A. N. Kirillov, Yang-Baxter equation, symmetric functions, and Schubert polynomials, Discrete Mathematics 153, (1996) 123-143. MR 98b:05101

[4] S. Fomin and R. Stanley, Schubert polynomials and the nil-Coxeter algebra. Adv. Math. 103 (1994), no. 2, 196-207. MR 95f:05115

[5] M. Kogan RC-graphs and Littlewood-Richardson Rule. Internat. Math. Res. Notices. 2001, no. $15,765-782$.

[6] A.Lascoux and M. P. Schutzenberger, Polynomes de Schubert, C.R.Acad. Sci. Paris 294 (1982) 447-450. MR 83e:14039

[7] A.Lascoux and M. P. Schutzenberger, Schubert polynomials and the Littlewood-Richardson Rule, Let. Math. Phys. 10 (1985) 111-124. MR 87g:20021

[8] I. G. Macdonald, Notes on Schubert Polynomials, Publications du LACIM 6, Université du Québec à Montréal, 1991.

[9] L. Manivel. Fonctions symétriques, polynômes de Schubert et lieux de dégénérescence. Cours Spécialisés, No. 3, Société Mathématique de France, Paris, 1998. MR 99k:05159

[10] Alexander Postnikov, On a quantum version of Pieri's formula, Advances in Geometry (J.L. Brylinski et al., eds.), Progr. Math., vol. 172, Birkhäuser, Boston, 1999, pp. 371-383. MR 99m:14096

[11] F. Sottile, Pieri's formula for flag manifolds and Schubert polynomials, Annales de l'Institut Fourier 46 (1996), 89-110. MR 97g:14035

[12] Fulton, W., Young tableaux : with applications to representation theory and geometry, Cambridge University Press, 1997. MR 99f:05119

[13] R. Winkel. A combinatorial bijection between Standard Young Tableaux and reduced words of Grassmannian permutations. Sem. Loth. Comb. 36 (1996), Art. 536h. MR 98a:05157

Department of Mathematics, Northeastern University, Boston, Massachusetts 02115

E-mail address: misha@research.neu.edu

Department of Mathematics, Massachusetts Institute of Technology, Cambridge, MASSACHUSETTS 02139

E-mail address: abhinavk@mit.edu 